\title{
The Disambiguation and Application of the English Syntactic Ambiguity
}

\author{
Lijun Yang \\ School of Business and Trade \\ Huanghe S\&T University \\ Zhengzhou, China \\ e-mail:379630960@qq.com
}

\begin{abstract}
English ambiguity is an intrinsic phenomenon of language itself and exists widely. English ambiguity mainly includes the semantic ambiguity caused by polysemous words and sentences of syntactic ambiguity which may contain different understanding at the aspect of structures. Syntactic ambiguity expresses rich connotation with the limited notations, and therefore it has been paid great attention in the linguistic study. On the one hand, the syntactic ambiguity has negative effect, which causes the human communication to produce the misunderstanding; on the other hand, it also has the positive function. The strengthened subject, the vivid and humorous effect may be obtained as long as appropriate application of syntactic ambiguity. So through the analysis of the syntactic ambiguity, we may improve the abilities of English comprehension, and thus the ability of understanding and applying English language may be improved. This article first analyzes the categories and causes of syntactic ambiguity, and then analyzes the disambiguation and application of the English syntactic ambiguity.
\end{abstract}

Keywords-English syntactic; ambiguity; disambiguation application

\section{INTRODUCTION}

The natural resource of human language to express the thinking is limited, but human thinking is unlimited, thus there is ambiguity. The ambiguity may possibly hinder the human communication and people may avail the ambiguity to produce the humorous effect and improve the communication. So through the phenomenon of ambiguity, the categories and causes of ambiguity can be understood well, the efficient method of the disambiguation and application of the English ambiguity can be found, and then we can better understand and apply correctly the language. The ambiguity mainly includes phonetic ambiguity, vocabulary ambiguity and syntactic ambiguity. The syntactic ambiguity is the key point of grammatical research, which refers to the phenomenon that the sentence contains many kinds of meanings for the combination of different parts of component, and generally the ambiguity may be considered as one characteristic of the sentence.

\section{CAUSES OF ENGLISH SYNTACTIC AMBIGUITY}

We should first understand clearly the causes of it.

\section{A. Syntactic Ambiguity Caused by the Denial Scope}

There is a great discrepancy of conveying the denial methods between English Chinese. In English, the negative adverb "not" causes the syntactic ambiguity frequently.

Example 1:

They did not come to see her.

"not" may modify "come" and indicate that they completely have not come to see her; or may also modify "to" and indicate that they come not to see her.

\section{B. Syntactic Ambiguity Caused by Words' Special Syntactic Function}

English conjunctions, adverbs and pronouns which introduce subordinate clauses have many kinds of syntactic functions. When the subordinate clause introduced by them lies at the end of the sentence, and the predicate of the principal clause is an intransitive verb or a transitive verb which may be followed by the object or the double object. The subordinate clause possibly is the adverbial subordinate clause, and also it may be the object subordinate clause acting as direct object and also perhaps an attributive clause.

Example 2:

They will tell me when she comes.

This sentence may be transformed into the following two phrases: "When she comes, they will tell me." (Adverbial subordinate clause) or "They will tell me what time she comes (object subordinate clause)

\section{Syntactic Ambiguity Caused by Improper Abbreviation}

In English improper abbreviation may cause the ambiguity frequently.

\section{Example 3:}

He likes horses more than Jane.

For the same predicate verb "likes" as principal clause has been abbreviated after "Jane" and "Jane" may be regarded as the pronoun of the nominative case or the objective case, and then this phrase has two different meanings: "She likes horses 
more than she likes Jane." or "She likes horses more than Jane likes horses."

\section{Syntactic Ambiguity Caused by the Unclear Characteristics of Words}

Certain English words have two characteristics of the noun and the adjective, when these words serve as the attribute, ambiguity is easy to produce.

\section{Example 4:}

Drinking water should be moderate.

The ambiguity in this sentence comes from the phrase "drinking water". Because there are two kinds of syntactic explanations: First, "drinking" will be regarded as one present participle, and it explains the condition of the "water", "water" is modified by "drinking", "drink" is a intransitive verb; Second, we may treat the "drinking" as the gerund, and namely "water" may be regarded as the logical object of the "drinking"; "drink" as a transitive verb, and the entire sentence's subject is this movement of "drinking water", but is not the "water". Therefore the ambiguity exists in this phrase.

\section{E. Syntactic Ambiguity Caused by the Unclear Relations of Modifier}

If the relations of modifier are not clear, the syntactic ambiguity can thus produce.

\section{Example 5:}

The teacher praised the student with a smile.

This preposition phrase "with a smile" may be taken as the attribute of "the student", it means "The teacher praised the student who carried a smile", the preposition phrase "with a smile" might also be taken as the way adverb, it means "The teacher praised the student by the way of a smile." It is one of the syntactic functions of the adjectives that act as the attribute. It can compose a modifier together with a noun to modify a noun; the adjective may also be followed by two connecting compound nouns; it may also modify a noun together with another adjective, which constitutes dual modifying relations. The kind of ambiguity is ambiguity of structure on the surface.

\section{DISAMBIGUATION OF THE ENGLISH SYNTACTIC AMBIGUITY}

From the above discussion, we may understand that English syntactic ambiguity is a kind of common and complex phenomenon. Moreover the negative influence will inevitably come into being as well, and thus many inconveniences are brought to our communication. In the process of our verbal communication, we should as far as possible avoid or eliminate the phenomenon of syntactic ambiguity which causes the inconvenience and avoid the misunderstanding. We may adopt the following several efficient paths to make the disambiguation:

\section{A. Adoption of the Punctuation Marks}

We often use the methods of rewriting or replenishing punctuation marks to indicate clearly your meaning of expression and eliminate the ambiguity.

Example 6:

"Jack beat Mr. Wang." refers to: Jake has hit Mr. Wang. "Jack beat Mr. Wang?" means: Dose Jake hit Mr. Wang? "Jack, beat Mr. Wang." Namely: Jake, hit Mr. Wang.

Example 7:

"Don't write anything." means anything should not be written. "Don't write-anything." means you should not write anything any more.

Example 8:

It's a pretty small island.

This phrase contains: It is a quite small island; it is a beautiful small island. But the sentence: "it's a pretty, small island." has one meaning: That is a beautiful island.

Example 9:

Be good children

It means: Do a good child! Or the children must be good! The sentence: "Be good, children" refers to the latter.

Moreover, when the sentence's predicate verb is the negative form followed by an adverbial subordinate clause, the ambiguity often come out easily.

Example 10:

I did not go because 1 was afraid.

This sentence may be understood as: I have not gone, because I am afraid; I go and not because I am afraid.

\section{B. Change of Vocabulary Collocation and Order}

Regarding the ambiguity caused by polysemy, it may be eliminated by changing the vocabulary collocation.

\section{Example 11:}

What is his position?

"Position" will cause the ambiguity of the sentence. Namely it includes two kinds of meaning: the manner or the place. If "his manner" is asked for, "attitude" may be used; if "his place" is asked for, "where" may be adopted. So we can express this phrase as follows: What is his attitude? Or where is his position? Also we can replace the possessive noun with the "of phrase" to make the disambiguation.

\section{Complement of the Omitted Parts}

The syntactic ambiguity caused by the omitted parts of the comparative structure may be avoided through complementing the omitted parts which are easy to be misunderstood.

Example 12:

He likes cars as much as his daughter. 
We can complement the phrases as "He likes cars as much as he likes his daughter." or "He likes cars as much as his daughter does."

\section{Use of the Stress and Stop of Spoken Language}

We may avail stress and stop of spoken language to eliminate the ambiguity.

\section{Example 13:}

You must answer all the questions on the paper.

If the speaker does not emphasize specially, the listener will feel puzzled: what on earth it refers to "replying all the questions of the paper" or "answering all the questions on the paper"? If the speaker stresses "all the questions" or "on the paper", the ambiguity may be avoided. Read aloud may also achieve the goal of reducing the ambiguity.

Example 14:

Did you read that letter to the editor?

It may be read as "Did you read/that letter to the editor?" (Did you read that letter for the editor?) Or "Did you read that letter/to the editor?" (Do you have the editor listen that letter?)

\section{E. Change of the Structure of Sentences}

First, the prepositional phrase may be taken as the adverbial or the attribute in the sentence and may easily produce the ambiguity. If we bring forward the prepositional phrase of the former or change the prepositional phrase of the latter into the attributive clause, the ambiguity can be eliminated.

\section{Example 15:}

John washed the car in the garage.

It may be changed into "In the garage, John washed the car." or "John washed the car which was in the garage." Second, we may add logical subject to "too...to" or change it into "so...that".

\section{Example 16:}

The lamb is too hot to eat.

We may use the following structure to avoid the ambiguity separately: "The cooked lamb is too hot for someone to eat." (This mutton is too hot to be unable to eat.) Or "The lamb is so hot that it cannot eat anything. (Lamb feels very hot and cannot eat any thing.) Third, change the "ing" to "to do" or attributive clause.

\section{Example 17:}

Visiting cousins can be a nuisance.

The "Visiting cousins" may indicate "To visit cousins" (visit cousins/sisters) or also indicate "cousins that visit us" (cousins/sisters who visit us). If we adjust the structure of sentence, the syntactic ambiguity may be eliminated. We can adjust the phrases as: "It can be a nuisance to visit cousins". Or "The cousins that visited us could be a nuisance."

\section{F. Dependence on Linguistic Environment}

The linguistic environment can be divided into the linguistic environment of language and the linguistic environment of scene roughly (Zhang Ning, 2001:90). The linguistic environment of language refers to matching possibility of words or phrases; the linguistic environment of scene refers to the background of characters, society, culture and politics concerning the communication. In daily communication the linguistic environment of scene also includes eyesight, facial expression and gesture etc.

Example 18:

Afghanistan's occupation.

It refers to "the Afghanistan occupied other countries", or "other countries (for example the former Soviet Union or the US) occupied Afghanistan" The ambiguity has come out because the use of the possessive form. If we knew that in 2001 this linguistic environment of the US attacked Afghanistan Taliban regime, the ambiguity can be eliminated naturally.

\section{Example 19:}

Who is the woman next to the man reading a newspaper?

The "reading a newspaper" refers to "the woman" or "the man"? If there will be concrete linguistic environment, we can understand the phrase clearly.

\section{APPLICATION OF THE ENGLISH SYNTACTIC AMBIGUITY}

Although English syntactic ambiguity hinders the people's understanding of the words in a certain degree, and the difficulty of human communication has been increased, the syntactic ambiguity also has its positive role and should widely been applied in such kinds of situation as the daily communication, literary works, commercial advertisement and so on to achieve some specific rhetoric effect or the goal of pragmatics.

\section{A. Syntactic Ambiguity is Applied in the Advertisement}

In the advertisement, the homonym is often used to draw people's attention and produce the humorous effect. Homonym with the same form refers to those words with the same form and different meaning. Some advertising agents fully use the homonym with the same form in order to attract customers.

\section{Example 20:}

Sur Prize, a pun to surprise you with the prize.

Here "surprise" has double meanings, namely one is its original meaning, and the other is the sum of Sur and Prize. So it is easy to draw customers' attention.

\section{Example 21:}

We have courses to make grown man young and a young man the groan.

Here humorous effect just has used the same pronunciation to produce the intentional ambiguity, because 
both "grown" and "groan" are a pair of homonym, they are used ingeniously in the identical sentence, and thus humor comes out naturally.

\section{Example 22:}

Spoil yourself and not your figure.

This is an advertisement of an ice cream company. Here "spoil" is an ambiguous word, "spoil oneself" means "enjoy heartily"; but "spoil one's figure" means "damages person's figure". In this advertisement the ambiguous sentence is used to emphasize a promise from the manufacturers to the consumers: This kind of ice cream can let you luckily enjoy the delicious dishes, but it will not harm your figure in fact. It has catered to those dieters' psychological demand exactly and let them accept this product happily. So, if we use well the homonym, good expressive effect will be obtained.

\section{B. Syntactic Ambiguity is Applied in the Literary Works}

The syntactic ambiguity applied widely in the works may be used well to lay stress on the characters' dispositions, and then the fascinating story may be developed well.

\section{Example 23:}

Ask for me tomorrow, and you shall find me a grave man.

In the drama "Romeo and Juliet" of Shakespeare, one character has received a fatal sword, on his deathbed he said those words. In the sentence "a grave man" may be understood as "a person in a grave" or "a solemn and serious person". These two kinds of meaning are contained in the phrases and both of them are hard to be eliminated. Just because this kind of ambiguous expression, the spirit taking death calmly as well as humorous and optimistic disposition of the character is expressed very correctly and vividly.

\section{Syntactic Ambiguity is Applied in the Speech}

A pun on the word may strengthen the subject of the speech and make the speech more brilliantly.

\section{Example 24:}

We must all hang together, or we shall all hang separately. (We must unite; otherwise any of us will be strangled).

This is a famous pun on the word said by the renowned statesman Benjamin. Franklin in 1746. The statesman had made use of "hang" ingeniously which contained two different significances to express the subject: "the unity is the strength", the words' strength is strengthened, the audiences or the readers are be made the profound impression on, and thus the effect of political propaganda had been guaranteed.

\section{Syntactic Ambiguity Applied in the Communication}

Besides the advertisement, we can also avail the homonym to express meaning tactfully when we communicate, and in this way a good and happy atmosphere of communication may be obtained; the meaning will be easier to be accepted as well as the intention of the speaker may be achieved easily.

\section{Example 25:}

"You are not eating your fish", the waitress said to him. "Anything wrong with it?" "Long time no sea." The man replied.

"Sea" and "see" are a group of antonym. This customer did not want to say "they don't meet with each other very long time", but expressed wittedly the fish was not fresh.

\section{Example 26:}

"This is a white hote1." He said. I looked around "It is not white. Such a color needs a great deal of cleaning." I said, "But I do not think I mind."

The shop owner wished obviously to express the hotel was provided for the Caucasian, but not the Black, "I" actually intentionally understood "the white hotel" and further pointed out that this shop was not very clean, but "I pay no attention to it". Obviously, "I" shifted the topic to the advantageous direction towards him and expresses the meaning tactfully.

\section{Example 27:}

"My daughter has arranged a little piece for the piano.", "Good, it's about time we had a little peace."

The master wanted to let the daughter play the piano for the visitor, but the customer actually wanted to rest a while. Because "piece" and "peace" have the same pronunciation, thus it cause the customer's misunderstanding and produce completely irrelevant answers to the master and caused the people to feel high.

\section{CONCLUSION}

Generally speaking, the syntactic ambiguity prevents the communication from going on smoothly; we must avoid this kind of ambiguity as far as possible. But the syntactic ambiguity may be taken as one kind of common phenomenon in the natural language, there must be the reason. Although the syntactic ambiguity will affect the human communication, it will produce the unexpected rhetoric results, especially in the advertisement, literary works and so on. There are many reasons which result in English syntactic ambiguity; however, even if we have understood all the reasons, the syntactic ambiguity may be not avoided completely. In order not to cause the syntactic ambiguity to influence human communication, we must unify the concrete linguistic environment to analyze and judge the meaning of sentences as far as possible. Moreover, it is not necessary for us to eliminate the syntactic ambiguity completely, because sometimes the syntactic ambiguity will produce the positive effect, such as satire, humor, and emphasis. In brief, in the process of studying or using English, we should treat the phenomenon of syntactic ambiguity dialectically.

\section{ACKNOWLEDGMENT}

I would like to give my heartfelt thanks to many people for their help in my academic studies over the past years. My deepest gratitude to my family, thanks for their kindness, patience, encouragement. Thanks to my school that has give 
me this delicious opportunity. And thanks to my dear friends who have given their great help to me in my research.

\section{REFERENCES}

[1] Cruse D. A. Lexical Semantics[M]. Cambridge: Cambridge University Publisher, 1986.

[2] Gil Brown. Language and Understanding[M]. Shanghai: Shanghai Foreign Language Education Press, 1994.

[3] GuKui. Analysis of English Syntactic Ambiguity [J]. Journal of AnHui Water Conservancy and Hydropower Vocation Technology College, 2006, (6).

[4] LiChang. Analysis of English Ambiguity[J]. Journal of LiaoNing Science and Technology College, 2007,(9).

[5] LiMingqing. Effective Methods of Disambiguation English Ambiguity[J]. Journal of HuNan Commercial College, 2004, (11).

[6] YuanJun, Origin and Countermeasures of English Syntactic Ambiguity[J]. HuNan Education, 2005, (24). 